\title{
ON SUMMARY MEASURES ANALYSIS OF THE LINEAR MIXED EFFECTS MODEL FOR REPEATED MEASURES WHEN DATA ARE NOT MISSING COMPLETELY AT RANDOM
}

\author{
RODERICK J. LITTLE* AND TRIVELLORE RAGHUNATHAN \\ Department of Biostatistics and Institute for Social Research, University of Michigan, 1420 Washington Heights, Ann Arbor, \\ MI 48109-2029, U.S.A.
}

\begin{abstract}
SUMMARY
Subjects often drop out of longitudinal studies prematurely, yielding unbalanced data with unequal numbers of measures for each subject. A simple and convenient approach to analysis is to develop summary measures for each individual and then regress the summary measures on between-subject covariates. We examine properties of this approach in the context of the linear mixed effects model when the data are not missing completely at random, in the sense that drop-out depends on the values of the repeated measures after conditioning on fixed covariates. The approach is compared with likelihood-based approaches that model the vector of repeated measures for each individual. Methods are compared by simulation for the case where repeated measures over time are linear and can be summarized by a slope and intercept for each individual. Our simulations suggest that summary measures analysis based on the slopes alone is comparable to full maximum likelihood when the data are missing completely at random but is markedly inferior when the data are not missing completely at random. Analysis discarding the incomplete cases is even worse, with large biases and very poor confidence coverage. Copyright (C) 1999 John Wiley \& Sons, Ltd.
\end{abstract}

\section{INTRODUCTION}

Many longitudinal studies suffer from attrition, that is, subject dropping out prematurely. Examples include panel surveys or cohort studies, and clinical trials with designs that involve repeated measurements to chart the course of a disease. The resulting data are unbalanced with unequal numbers of measures for each subject. Mixed linear effects models with normal errors ${ }^{1-3}$ provide a flexible tool for analysing unbalanced longitudinal data, and software for maximum likelihood estimation under these models is now widely available to practitioners. ${ }^{4,5}$ These analysis tools are valuable in that they incorporate all the available information in the data, and they can reduce or even eliminate the bias resulting from an analysis confined to the complete cases. However, estimates from these models assume that the missing data are missing at

\footnotetext{
* Correspondence to: Roderick J. Little, Department of Biostatistics and Institute for Social Research, University of
} Michigan, 1420 Washington Heights, Ann Arbor, MI 48109-2029, U.S.A. E-mail: rlittle@umich.edu

Contract/grant sponsor: National Science Foundation

CCC 0277-6715/99/182465-14\$17.50

Copyright (C) 1999 John Wiley \& Sons, Ltd. 


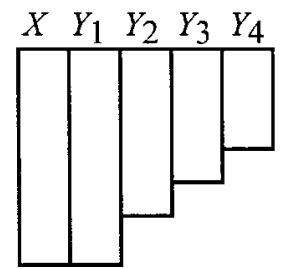

Figure 1. Schematic of a monotone missing data pattern, with $X$ representing covariates, $Y_{1}, \ldots, Y_{4}$ repeated measures at four time points, and blocks representing data

random, in Rubin's ${ }^{6}$ sense. A number of methods that provide for drop-out processes that are not missing at random have also been proposed. ${ }^{7-15}$ Little $^{16}$ reviews these methods within a unified framework based on likelihood inference for models for the data and the drop-out mechanism.

A drawback of these approaches is that they require specification of a full model for the vector of repeated measures for each individual, and a model for the drop-out mechanism if it is not missing at random. A simple and intuitive alternative approach is summary measures analysis; one or more measures of interest are formulated, these measures are estimated for each subject from their set of repeated measures, and the resulting summary measures are then regressed on subject-level characteristics. This approach may be less efficient than an analysis based on a full statistical model for the repeated measures, but it has potential advantages in terms of simplicity and reduced modelling assumptions, and it is very commonly applied in practice. Naïve applications of the summary measures approaches do not take into account the fact that the measures have differential precision when based on variable numbers of repeated measures. We study here a relatively refined version of the approach that takes this differential precision into account in the context of a linear mixed effects model.

We compare the summary measures approach with inference based on a full model for the repeated measures ignoring the missing-data mechanism, using both maximum likelihood and the method of moments to estimate the variances. Our main interest is in comparisons when the data are not missing completely at random, in the sense that missingness depends on values of the repeated measures. For concreteness we consider linear models where the repeated measures are regressed on time, and the summary measures are an estimated slope and intercept for each individual. Specific questions we address are:

(a) What assumptions about the missing data mechanism are implied by the summary measures approach?

(b) What are the relative merits of multivariate and univariate summary measures analyses when the data are not missing completely at random?

(c) Under what circumstances is a full likelihood-based analysis based on a model for the vector of repeated measures preferable to a summary measures analysis? Are there practically interesting situations where the summary measures analysis dominates an analysis based on the full model?

(d) How important is it to estimate the variance parameters by maximum likelihood, rather than by simpler approaches based on the method of moments? 


\section{MODELS FOR THE REPEATED MEASURES AND DROP-OUT MECHANISM}

We adopt the following general model for repeated-measures data with drop-outs. ${ }^{16}$ Suppose that the data for subject $i$ consist of up to $K$ repeated measures on outcome variables $Y_{1}, \ldots, Y_{K}$. The data form a monotone pattern as displayed in Figure 1, so that if $Y_{j}$ is missing then $Y_{k}$ is missing for all $k>j$. For subjects $i=1, \ldots, n$, let $y_{i}=\left(y_{i 1}, \ldots, y_{i K}\right)$ be a $(1 \times K)$ complete-data vector of outcomes for subject $i$, possibly incompletely observed. We write $y_{i}=\left(y_{\mathrm{obs}, i}, y_{\mathrm{mis}, i}\right)$, where $y_{\mathrm{obs}, i}=$ observed part of $y_{i}, y_{\mathrm{mis}, i}=$ missing part of $y_{i}$. Note that lower-case $y_{i}$ denotes rows (cases) and upper-case $Y_{k}$ denotes columns (variables).

$X_{i}$ denotes fixed covariates or design matrices, including times of measurement $\left(t_{i 1}, \ldots, t_{i K}\right)$ measured from some meaningful baseline, for example, calendar time or start of treatment. These variables are assumed fully observed. $R_{i}$ is the missing-data indicator, indexing complete and incomplete patterns of data. Specifically, let $R_{i}=0$ for complete cases, and let $R_{i}=k$ if a subject drops out between the $(k-1)$ th and $k$ th observation time, that is, $y_{i 1}, \ldots, y_{i, k-1}$ are observed and $y_{i k}, \ldots, y_{i K}$ are missing. $\beta_{i}$ is the $(q \times 1)$ vector of unobserved random coefficients characterizing the mean of $y_{i} \cdot \gamma$ is the set of fixed model parameters for the distribution of $y_{i}$ and $\psi$ is the set of fixed model parameters for the distribution of $R_{i}$ given $y_{i}$.

We focus here on a special case of this model where the mean of $y_{i j}$ is a linear function of time with a random slope and intercept for each individual. That is

$$
\begin{gathered}
\left(y_{i j} \mid \beta_{i}, \gamma\right) \sim_{\text {ind }} \mathrm{N}\left(\beta_{i 0}+\beta_{i 1} t_{i j}, \sigma^{2}\right), \quad j=1, \ldots, K, \\
\beta_{i} \sim \mathrm{N}_{2}(\theta, \Lambda) ; \quad \Lambda=\left(\begin{array}{cc}
\tau_{0}^{2} & \rho \tau_{0} \tau_{1} \\
\rho \tau_{0} \tau_{1} & \tau_{1}^{2}
\end{array}\right)
\end{gathered}
$$

where $\mathrm{N}_{k}$ denotes the $k$-variate normal distribution, the random coefficients $\beta_{i}=\left(\beta_{i 0}, \beta_{i 1}\right)^{\mathrm{T}}$ are a random intercept and slope for subject $i, \theta=\left(\theta_{0}, \theta_{1}\right)^{\mathrm{T}}$ is the average intercept and slope and the set of fixed model parameters are $\gamma=\left(\theta_{0}, \theta_{1}, \sigma^{2}, \tau_{0}^{2}, \tau_{1}^{2}, \rho\right)$.

We consider models for the joint distribution of $y_{i}, R_{i}, \beta_{i}$ given $X_{i}$, assuming that given $X=\left(X_{1}, \ldots, X_{n}\right),\left(y_{i}, R_{i}, \beta_{i}\right)$ and $\left(y_{j}, R_{j}, \beta_{j}\right)$ are independent for $i \neq j$. Inference for the fixed parameters $\gamma$ of the model can be based on the observed likelihood of $\gamma$ given the data $\left\{\left(y_{\mathrm{obs}, i}, R_{i}\right): i=1, \ldots, n\right\}$, obtained formally by integrating the unobserved quantities $\left\{y_{\text {mis }, i}, \beta_{i}: i=1, \ldots, n\right\}$ out of the joint distribution of $\left\{y_{i}, R_{i}, \beta_{i} i=1, \ldots, n\right\}$ for the chosen model:

$$
L\left(\gamma, \psi \mid Y_{\text {obs }}, R\right)=\text { const } \times \prod_{i=1}^{n} \int p\left(y_{\text {obs }, i}, y_{\text {mis }, i}, R_{i}, \beta_{i} \mid X_{i}, \gamma, \psi\right) \mathrm{d} y_{\text {mis }, i} \mathrm{~d} \beta_{i} .
$$

Large-sample inference for fixed parameters $\gamma$ can be based on the method of maximum likelihood with associated asymptotic standard errors, using iterative algorithms such as scoring, EM and extensions. ${ }^{17-19}$ Bayesian inference can be implemented via stochastic simulation, ${ }^{20-22}$ and provides a better analysis than maximum likelihood for small samples.

This approach requires specification of a model for the distribution of $R_{i}$ given $y_{i}, X_{i}$, which is often not an easy task. A simpler approach is to base inference on the likelihood ignoring the drop-out mechanism:

$$
L\left(\gamma \mid Y_{\text {obs }}\right)=\text { const } \times \prod_{i=1}^{n} \int p\left(y_{\text {obs }, i}, y_{\text {mis }, i}, \beta_{i} \mid X_{i}, \gamma\right) \mathrm{d} y_{\text {mis }, i} \mathrm{~d} \beta_{i} .
$$


Inference based on (3) is valid (although not necessarily fully efficient) when the drop-out mechanism is missing at random, ${ }^{6,16}$ in that it does not depend on the missing data $y_{\text {mis }, i}$ or unobserved random coefficients $\beta_{i}$ after conditioning on the observed variables $X_{i}, y_{\mathrm{obs}, i}$ and the parameters $\psi$ :

$$
p\left(R_{i} \mid X_{i}, y_{\mathrm{obs}, i}, y_{\mathrm{mis}, i}, \beta_{i}, \psi\right)=p\left(R_{i} \mid X_{i}, y_{\mathrm{obs}, i}, \psi\right) .
$$

If (4) holds and the parameters $\gamma$ and $\psi$ are also distinct, then inference based on (3) is efficient and equivalent to inferences based on the full likelihood (2). The drop-out mechanism is then called ignorable. ${ }^{6,16}$

We define two other classes of drop-out mechanisms, one stronger than missing at random and one weaker. As discussed in Little, ${ }^{16}$ a number of alternative methods to maximum likelihood, including analysis of the complete cases and unweighted forms of generalized estimating equations, make the stronger assumption of covariate-dependent drop-out, where missingness is allowed to depend only on the covariates $X_{i}$ :

$$
p\left(R_{i} \mid X_{i}, y_{\mathrm{obs}, i}, y_{\mathrm{mis}, i}, \beta_{i}, \psi\right)=p\left(R_{i} \mid X_{i}, \psi\right) .
$$

Some authors call this condition missing completely at random, ${ }^{13}$ but we adopt a terminology more consistent with Rubin's original ideas ${ }^{6}$ and confine the latter term to mechanisms that do not depend on the random effects, the repeated measures or the covariates. ${ }^{16}$

We also find it useful to distinguish mechanisms that are missing at random conditional on the values of the unknown random coefficients $\beta_{i}$, and accordingly propose the following new definition. The drop-out mechanism is called subject-specific missing at random if it does not depend on the missing data but is allowed to depend on the observed variables and unobserved random coefficients:

$$
p\left(R_{i} \mid X_{i}, y_{\mathrm{obs}, i}, y_{\mathrm{mis}, i}, \beta_{i}, \psi\right)=p\left(R_{i} \mid X_{i}, y_{\mathrm{obs}, i}, \beta_{i}, \psi\right) .
$$

Subject-specific missing at random is clearly weaker than missing at random. It is relevant in the repeated-measures setting since under this condition maximum likelihood-based summary measures calculated separately for each individual are consistent for the true individual value as the number of repeated measures on an individual tends to infinity. This form of asymptotics is unrealistic for small numbers of repeated measures but has some relevance for longer series of measurements.

We now confine attention to the model (1), and analyses that ignore the drop-out mechanism. We assume for simplicity that all cases have at least two observations. In that case, maximum likelihood estimates for the average slope and intercept under (1) can be expressed in terms of the least squares intercept and slope $\hat{\beta}_{i}=\left(\hat{\beta}_{i 0}, \hat{\beta}_{i 1}\right)^{\mathrm{T}}$ estimated from the observed vector of repeated measurements for subject $i$. Since the number of repeated measures for each subject varies, these estimates have differential precision. Under the model (1) we have

$$
\hat{\beta}_{i} \sim \mathrm{N}_{2}\left(\theta, V_{i}\right), \text { where } V_{i}=\left(\begin{array}{ll}
\tau_{0}^{2}+\sigma^{2}\left\{k_{i}^{-1}+\bar{t}_{i}^{2} u_{i}^{-1}\right\} & \rho \tau_{0} \tau_{1}-\sigma^{2} \bar{t}_{i} u_{i}^{-1} \\
\rho \tau_{0} \tau_{1}-\sigma^{2} \bar{t}_{i} u_{i}^{-1} & \tau_{1}^{2}+\sigma^{2} u_{i}^{-1}
\end{array}\right)
$$

and $\bar{t}_{i}$ and $u_{i}=\sum_{j=1}^{k_{i}}\left(t_{i j}-\bar{t}_{i}\right)^{2}$ are, respectively, the mean and sum of squares about the mean of the times of measurement for subject $i$. We consider four methods of analyses for the average slope $\theta_{1}$, distinguished by (a) whether the least squares slope and intercept for each individual is 
included in the analysis or the slope alone, and (b) whether the variance parameters are estimated by maximum likelihood or by the method of moments.

To derive the analyses that use the slopes and intercepts, note that the maximum likelihood estimate of $\theta$ for known $V_{i}$ weights the least squares estimates $\hat{\beta}_{i}$ according to their precision, that is

$$
\tilde{\theta}=\left(\sum_{i=1}^{n} V_{i}^{-1} \hat{\beta}_{i}\right)\left(\sum_{i=1}^{n} V_{i}^{-1}\right)^{-1}
$$

With $V_{i}$ unknown, ignorable maximum likelihood (IML) estimates are obtained by replacing the variance parameters $\left(\tau_{0}^{2}, \tau_{1}^{2}, \rho, \sigma^{2}\right)$ in $V_{i}$ by maximum likelihood estimates, obtained by an iterative method such as scoring or EM. A simpler approach is to replace the maximum likelihood estimates of the variance parameters by non-iterative method of moment estimates ${ }^{23}$

$$
\tilde{\Lambda}=\frac{1}{n-1} \sum_{i=1}^{n}\left(\hat{\beta}_{i}-\bar{\beta}\right)\left(\hat{\beta}_{i}-\bar{\beta}\right)^{\mathrm{T}}-\frac{\hat{\sigma}^{2}}{n} \sum_{i=1}^{n}\left(\begin{array}{cc}
\left\{k_{i}^{-1}+\bar{t}_{i}^{2} u_{i}^{-1}\right\} & -\bar{t}_{i} u_{i}^{-1} \\
-\bar{t}_{i} u_{i}^{-1} & u_{i}^{-1}
\end{array}\right)
$$

where $\bar{\beta}$ is the unweighted average of the $\hat{\beta}_{i}$. We call the resulting method generalized least squares (GLS), following Wang-Clow et al. ${ }^{24}$

If interest is in the average slope and the intercept is a nuisance parameter, a univariate summary measures approach discards the estimated intercepts and bases inference on the individual slopes $\hat{\beta}_{i 1}$ alone, together with a pooled estimate of $\sigma^{2}$. For known $V_{i}$, the maximum likelihood estimate of the slope based on these data is

$$
\breve{\theta}_{1}=\left(\sum_{i=1}^{n}\left(\tau_{1}^{2}+\sigma^{2} u_{i}^{-1}\right)^{-1} \hat{\beta}_{i 1}\right)\left(\sum_{i=1}^{n}\left(\tau_{1}^{2}+\sigma^{2} u_{i}^{-1}\right)^{-1}\right)^{-1} .
$$

The residual variance $\sigma^{2}$ is estimated by the pooled mean square $\hat{\sigma}^{2}=\sum_{i=1}^{n} \operatorname{rss}_{i} /(N-2 n)$ where rss $_{i}$ is the residual sum of squares from the regression for individual $i, N$ is the total number of observations and $n$ is the number of subjects. The between-subjects variance $\tau_{1}^{2}$ is estimated by its iterative maximum likelihood estimate conditional on $\sigma^{2}=\hat{\sigma}^{2}$. We call the resulting approach summary measures maximum likelihood (SMML). Estimates and large sample standard errors are readily computed using a maximum likelihood program such as SAS Proc Mixed. ${ }^{5}$ More simply, estimating $\tau_{1}^{2}$ by the method of moments

$$
\tilde{\tau}_{1}^{2}=\frac{1}{n-1} \sum_{i=1}^{n}\left(\hat{\beta}_{i 1}-\bar{\beta}_{1}\right)^{2}-\frac{\hat{\sigma}^{2}}{n} \sum_{i=1}^{n} u_{i}^{-1}
$$

we obtain weighted least squares (WLS) estimates, again using the terminology in Wang-Clow et al. ${ }^{24}$

An even simpler approach is to discard the incomplete cases and base inferences on the complete cases. All the methods described above are equivalent when applied to the subset of complete cases, and we call the resulting method complete-case (CC) analysis.

These five methods - IML, GLS, SMML, WLS, CC analysis - yield asymptotically valid inferences when the missing data mechanism is missing completely at random, in that drop-out does not depend on the repeated measures or the unobserved random effects. The efficiency of the estimates varies, however. When data are not missing completely at random, the methods vary 
not only in relative efficiency but also in the degree of bias, as the simulation study described below indicates.

It is well known that IML yields valid inferences when the drop-out mechanism is missing at random, as in equation (4). CC analysis is consistent under covariate-dependent drop-out (5), but is generally biased when drop-out depends on the underlying random coefficients or the repeated measures. The degree of bias depends on the specific form of the mechanism. The method appeared competitive with IML in the simulation of Wang-Clow et al., ${ }^{24}$ suggesting that gains in methods that use the incomplete cases may be minor. However, the simulations reported here suggest that this finding was a consequence of the particular choices of parameters in that simulation, and does not have broad validity.

SMML is a maximum likelihood technique, so one might think it is valid for drop-out mechanisms that are missing at random. However, that is only true for maximum likelihood methods like IML that use all the data. For maximum likelihood methods that use only partial summaries of the data, the relevant assumption is that drop-out does not depend on the missing values after conditioning on the observed data in each case that are included in the analysis, that is, the summary measure for each case. Since the summary measure is a different combination of the repeated measures for each missing-data pattern, this condition reduces to the stronger assumption of covariate-dependent drop-out (5). To see this, we can write the pertinent missing at random assumption for the summary measures analysis as

$$
p\left(R_{i}=j \mid X_{i}, y_{\mathrm{obs}, i}, y_{\mathrm{mis}, i}, \beta_{i}, \psi\right)=p\left(R_{i}=j \mid X_{i}, c_{j}^{\mathrm{T}} y_{i}, \psi\right)
$$

where $c_{j}$ is the vector of coefficients defining the summary measure for the pattern $R_{i}=j$. In particular, for the SMML method $c_{j}^{\mathrm{T}} y_{i}=\hat{\beta}_{i 1}$. Summing the probabilities over all $J$ patterns yields

$$
\sum_{j=1}^{J} p\left(R_{i}=j \mid X_{i}, c_{j}^{\mathrm{T}} y_{i}, \psi\right)=1 \text { for all } y_{i} \text { and } X_{i} .
$$

Consider this constraint as $y_{i}$ and $X_{i}$ ranges continuously over all their possible values. Since the vector $c_{j}$ is different for each pattern $j$, the constraint implies that each $p\left(R_{i}=j \mid X_{i}, c_{j}^{\mathrm{T}} y_{i}, \psi\right)$ must be a constant function of its argument $c_{j}^{\mathrm{T}} y_{i}$, implying a covariate-dependent drop-out mechanism (5).

This argument does not prove that covariate-dependent drop-out is a necessary condition for SMML to yield consistent estimates, since Rubin's theory ${ }^{6}$ shows that the missing at random assumption is sufficient to ignore the mechanism, not necessary. The size of bias of SMML for particular missing-at-random mechanisms is not clear, and it is also of interest to study the properties of IML and SMML for non-MAR mechanisms. It seems possible that SMML could have less bias than IML if the estimated intercepts for each case that are input into the IML method are biased by the mechanism, but the estimated slopes are relatively free of bias.

The GLS and WLS methods can be expected to be more vulnerable to deviations from missing completely at random than their maximum likelihood counterparts IML and SMML, since the method-of-moments estimates of the variance parameters are not consistent under mechanisms other than covariate-dependent drop-out. However, GLS and IML, and WLS and SMML, have not been studied side by side in previous simulations, so the quantitative effects of using moment estimators rather than maximum likelihood estimators of the variance parameters have not been assessed. 

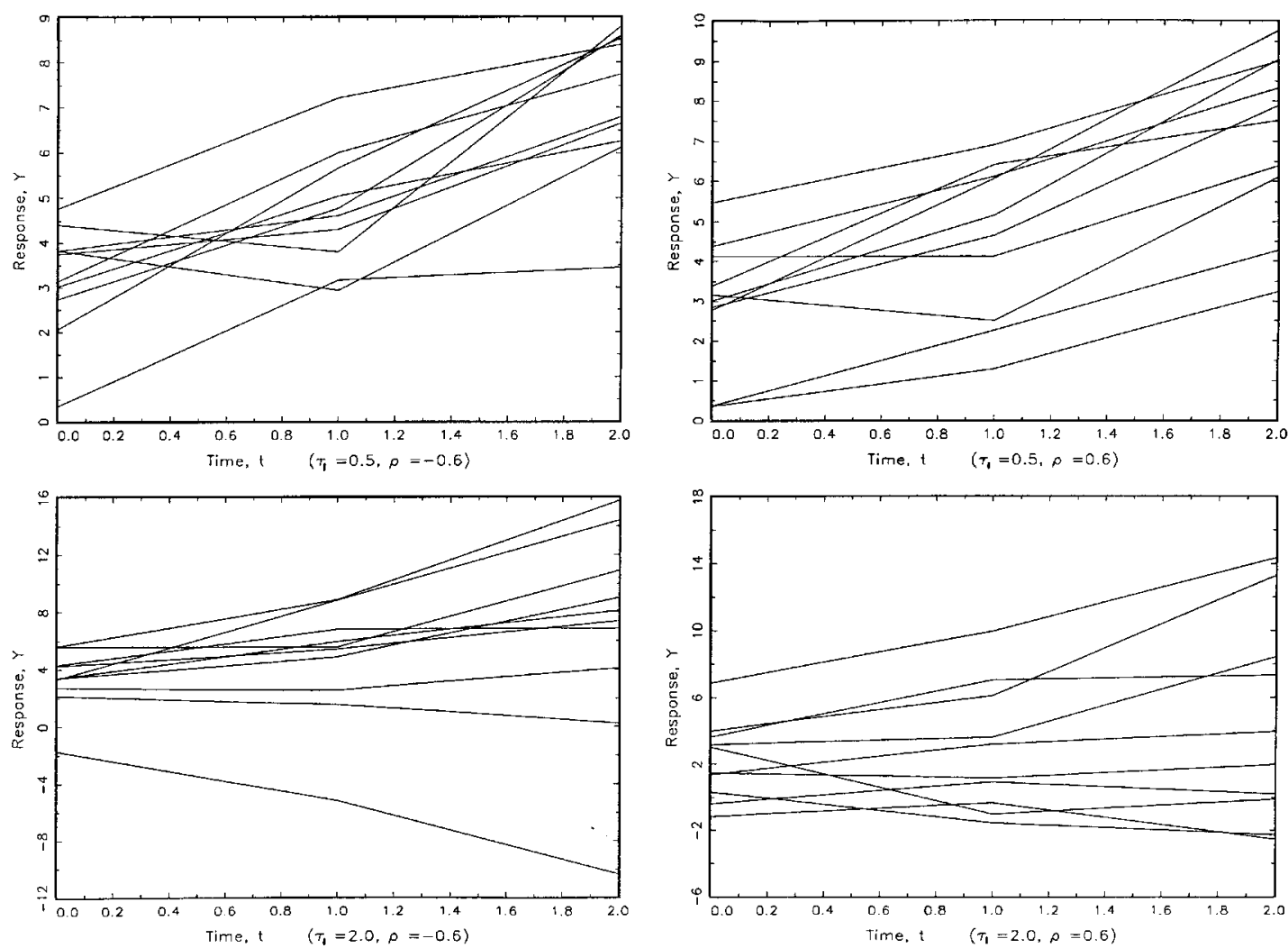

Figure 2. Profile plots of ten randomly chosen subjects from each of the four simulated populations

A simulation study was conducted to address these questions, under a broader range of drop-out mechanisms than have been considered in earlier studies.

\section{SIMULATION STUDY}

The five methods (IML, GLS, WLS, SMML, CC) were implemented on incomplete data sets with three time points and missing values confined to the third time point. That is, the observed data had only two patterns: complete cases and cases with the third observation missing. The complete data were generated using the following model. First, the subject-specific regression coefficients were generated as

$$
\left[\begin{array}{l}
\beta_{i 0} \\
\beta_{i 1}
\end{array}\right] \sim \mathrm{N}\left(\left[\begin{array}{l}
1 \\
2
\end{array}\right],\left[\begin{array}{cc}
1 & \rho \tau_{1} \\
\rho \tau_{1} & \tau_{1}^{2}
\end{array}\right]\right)
$$

then $n$ observations were generated independently from univariate normal distributions $\left[y_{i j} \mid \beta_{i 0}, \beta_{i 1}\right] \sim \mathrm{N}\left(\beta_{i 0}+\beta_{i 1} j, \sigma^{2}\right)$. Four sets of parameter values were generated according to a factorial design with $\sigma^{2}=4, \tau_{1}^{2}=0 \cdot 25,4, \rho=-0 \cdot 6,0 \cdot 6$. For each parameter set 1000 data sets were generated for sample sizes $n=200$ and 1000, yielding 8000 data sets in all. Figure 2 displays 
data for samples of ten individuals for each of the combinations of $\sigma^{2}, \tau_{1}^{2}$ and $\rho$, to give a sense of what the data look like. For each data set, missing values at the third time point were created by each of the following six drop-out mechanisms:

$\begin{array}{ll}\text { (MCAR) } & \operatorname{pr}\left[y_{i 3} \text { missing } \mid y_{i}, \beta_{i}\right]=\Phi\left\{\lambda_{0}\right\} \\ \text { (MAR-SUM) } & \operatorname{pr}\left[y_{i 3} \text { missing } \mid y_{i}, \beta_{i}\right]=\Phi\left\{\lambda_{0}-\lambda_{1}\left(y_{i 1}+y_{i 2}\right)\right\} \\ \text { (MAR-DIFF) } & \operatorname{pr}\left[y_{i 3} \text { missing } \mid y_{i}, \beta_{i}\right]=\Phi\left\{\lambda_{0}-\lambda_{1}\left(y_{i 2}-y_{i 1}\right)\right\} \\ \text { (NI-Intercept) } & \operatorname{pr}\left[y_{i 3} \text { missing } \mid y_{i}, \beta_{i}\right]=\Phi\left\{\lambda_{0}-\lambda_{1}\left(\beta_{i 0}+y_{i 1}-y_{i 2}\right)\right\} \\ \text { (NI-Slope) } & \operatorname{pr}\left[y_{i 3} \text { missing } \mid y_{i}, \beta_{i}\right]=\Phi\left\{\lambda_{0}-\lambda_{1}\left(\beta_{i 1}+y_{i 2}-y_{i 1}\right)\right\} \\ \text { (NI-Y3) } & \operatorname{pr}\left[y_{i 3} \text { missing } \mid y_{i}, \beta_{i}\right]=\Phi\left\{\lambda_{0}-\lambda_{1} y_{i 3}\right\}\end{array}$

where $\Phi$ is the cumulative normal distribution function. The coefficients $\lambda_{0}, \lambda_{1}$ in the above mechanisms were chosen so that approximately 30 per cent of cases have the third time point missing. The first mechanism is missing completely at random, which is equivalent to covariate-dependent drop-out for this model. The next two mechanisms are missing at random, where in MAR-SUM drop-out depends on the sum of the first two observations and in MAR-DIFF drop-out depends on the difference; the fourth and fifth mechanisms are non-ignorable (NI) elaborations of MAR-SUM and MAR-DIFF where missingness is also allowed to depend on the underlying unobserved intercept (NI-Intercept) or the underlying unobserved slope (NI-Slope); and the last mechanism NI-Y3 is non-ignorable since missingness depends on the outcome at the third repeated measure, which is missing for some cases. All the mechanisms except NI-Y3 are subject-specific missing at random as defined by equation (6).

One reason for studying data sets with just three time points is that the individual estimated intercepts and slopes are easily computed and interpreted. In particular

$$
\hat{\beta}_{i 1}= \begin{cases}y_{i 2}-y_{i 1}, & \text { for incomplete cases } \\ \left(y_{i 3}-y_{i 1}\right) / 2, & \text { for complete cases. }\end{cases}
$$

Table I displays the mean empirical bias and empirical standard deviation of the estimated average slopes from each the five methods, calculated over the 1000 data sets, for each parameter set and drop-out mechanism. Table IA shows results for the mechanisms that are missing completely at random and missing at random, and Table IB shows results for the non-ignorable mechanisms. The column label 'Cov' shows the number of 90 per cent confidence intervals that cover the true parameter value. These intervals are based on large-sample standard errors and a normal reference distribution, which are good approximations for the samples sizes simulated. If the intervals have correct nominal coverage we expect 900 to cover the true parameter value. The following conclusions can be drawn from this table:

(a) When data are missing completely at random, there is no evidence of bias for any of the methods, and all the methods have close to nominal coverage, as theory predicts. The CC method is less efficient than the other method when $\tau_{1}^{2}$ is large, reflecting the fact that there is more information in the incomplete cases in these settings. The other methods have very similar properties. In particular any loss of efficiency in the summary measures methods SMML and WLS is minor, and method of moments estimates of the variances parameters do not lead to noticeably inferior estimates of the average slope than the maximum likelihood estimates of the variance parameters. 
Table IA. Bias, standard deviation (SD) and confidence coverage of estimates of $\theta_{1}$ from five methods (missing completely at random and missing at random mechanisms)

\begin{tabular}{|c|c|c|c|c|c|c|c|c|c|c|c|c|}
\hline \multirow[t]{2}{*}{$N$} & \multirow[t]{2}{*}{$\rho$} & \multirow[t]{2}{*}{$\tau_{1}^{2}$} & \multirow[t]{2}{*}{ Method } & \multicolumn{3}{|c|}{ MCAR } & \multicolumn{3}{|c|}{ MAR-SUM } & \multicolumn{3}{|c|}{ MAR-DIFF } \\
\hline & & & & Bias & SD & $\mathrm{Cov}$ & Bias & SD & $\mathrm{Cov}$ & Bias & $\mathrm{SD}$ & $\mathrm{Cov}$ \\
\hline \multirow[t]{5}{*}{200} & \multirow[t]{5}{*}{$-0 \cdot 6$} & \multirow[t]{5}{*}{$0 \cdot 25$} & $\mathrm{CC}$ & -1 & 70 & 898 & -267 & 67 & 12 & 219 & 68 & 56 \\
\hline & & & WLS & 2 & 68 & 900 & 17 & 75 & 908 & -66 & 78 & 784 \\
\hline & & & SMML & 0 & 65 & 930 & -14 & 69 & 898 & -35 & 74 & 836 \\
\hline & & & GLS & 2 & 68 & 894 & 53 & 74 & 832 & -66 & 77 & 782 \\
\hline & & & IML & -1 & 70 & 904 & 9 & 74 & 894 & -8 & 76 & 892 \\
\hline \multirow[t]{5}{*}{200} & \multirow[t]{5}{*}{$0 \cdot 6$} & \multirow[t]{5}{*}{$0 \cdot 25$} & $\mathrm{CC}$ & -1 & 72 & 904 & -145 & 74 & 346 & 228 & 67 & 40 \\
\hline & & & WLS & 0 & 68 & 902 & 64 & 76 & 788 & -58 & 78 & 818 \\
\hline & & & SMML & 4 & 69 & 912 & 58 & 72 & 770 & -29 & 76 & 832 \\
\hline & & & GLS & 1 & 67 & 900 & 48 & 76 & 830 & -58 & 78 & 816 \\
\hline & & & IML & 2 & 67 & 912 & 19 & 73 & 894 & -21 & 72 & 896 \\
\hline \multirow[t]{5}{*}{200} & \multirow[t]{5}{*}{-0.6} & \multirow[t]{5}{*}{$4 \cdot 0$} & $\mathrm{CC}$ & 6 & 176 & 902 & -646 & 176 & 178 & 949 & 138 & 0 \\
\hline & & & WLS & 7 & 152 & 902 & 175 & 156 & 704 & -27 & 158 & 902 \\
\hline & & & SMML & 1 & 154 & 900 & 158 & 154 & 740 & -24 & 164 & 878 \\
\hline & & & GLS & 7 & 152 & 906 & 62 & 154 & 892 & -32 & 156 & 900 \\
\hline & & & IML & 6 & 159 & 892 & -7 & 150 & 908 & -4 & 152 & 904 \\
\hline \multirow[t]{5}{*}{200} & \multirow[t]{5}{*}{$0 \cdot 6$} & \multirow[t]{5}{*}{$4 \cdot 0$} & $\mathrm{CC}$ & -6 & 177 & 906 & 41 & 181 & 886 & 956 & 143 & 0 \\
\hline & & & WLS & -3 & 154 & 914 & 246 & 159 & 534 & -11 & 161 & 898 \\
\hline & & & SMML & -5 & 155 & 904 & 238 & 167 & 568 & -20 & 150 & 920 \\
\hline & & & GLS & -2 & 154 & 898 & 59 & 160 & 874 & -17 & 159 & 890 \\
\hline & & & IML & 12 & 148 & 908 & 12 & 152 & 918 & -17 & 162 & 904 \\
\hline \multirow[t]{5}{*}{1000} & \multirow[t]{5}{*}{$-0 \cdot 6$} & \multirow[t]{5}{*}{$0 \cdot 25$} & $\mathrm{CC}$ & 0 & 33 & 904 & -264 & 29 & 0 & 218 & 30 & 0 \\
\hline & & & WLS & -1 & 31 & 910 & 16 & 32 & 882 & -67 & 33 & 370 \\
\hline & & & SMML & -1 & 31 & 890 & -18 & 33 & 810 & -29 & 32 & 738 \\
\hline & & & GLS & -1 & 31 & 902 & 52 & 32 & 542 & -67 & 33 & 372 \\
\hline & & & IML & 0 & 30 & 892 & 1 & 30 & 912 & -5 & 33 & 899 \\
\hline 1000 & $0 \cdot 6$ & $0 \cdot 25$ & $\mathrm{CC}$ & 1 & 32 & 908 & -143 & 33 & 4 & 220 & 31 & 0 \\
\hline & & & WLS & 0 & 30 & 902 & 68 & 33 & 330 & -65 & 34 & 412 \\
\hline & & & SMML & 1 & 30 & 914 & 56 & 32 & 462 & -30 & 33 & 724 \\
\hline & & & GLS & 0 & 30 & 900 & 52 & 33 & 528 & -64 & 34 & 406 \\
\hline & & & IML & -3 & 30 & 912 & 18 & 29 & 898 & -15 & 33 & 896 \\
\hline 1000 & -0.6 & $4 \cdot 0$ & $\mathrm{CC}$ & 4 & 81 & 912 & -438 & 77 & 0 & 942 & 64 & 0 \\
\hline & & & WLS & -1 & 68 & 912 & 169 & 72 & 236 & -26 & 71 & 876 \\
\hline & & & SMML & 0 & 68 & 914 & 165 & 71 & 258 & -15 & 73 & 884 \\
\hline & & & GLS & -1 & 67 & 914 & 55 & 71 & 788 & -29 & 70 & 880 \\
\hline & & & IML & -3 & 70 & 880 & 7 & 68 & 908 & 13 & 68 & 908 \\
\hline 1000 & $0 \cdot 6$ & $4 \cdot 0$ & $\mathrm{CC}$ & 2 & 83 & 892 & 41 & 82 & 846 & 945 & 68 & 0 \\
\hline & & & WLS & 3 & 72 & 896 & 240 & 70 & 38 & -22 & 70 & 908 \\
\hline & & & SMML & -2 & 73 & 894 & 244 & 67 & 42 & -13 & 68 & 914 \\
\hline & & & GLS & 3 & 71 & 896 & 51 & 71 & 810 & -28 & 70 & 892 \\
\hline & & & IML & -8 & 70 & 900 & 16 & 72 & 892 & 1 & 69 & 899 \\
\hline
\end{tabular}

(b) For the MAR-SUM mechanism, IML is the best method, reflecting its large-sample optimality under this mechanism. It has no evidence of bias, precision that is generally better than the other methods, and close to nominal coverage. GLS exhibits moderate bias, reflecting the fact that the method of moments variance estimate is not consistent for mechanisms that are not missing completely at random. This bias translates into poor coverage, particularly for the large sample size. SMML performs comparably to IML when 
Table IB. Bias, standard deviation (SD) and confidence coverage of estimates of $\theta_{1}$ from five methods (non-ignorable mechanisms)

\begin{tabular}{|c|c|c|c|c|c|c|c|c|c|c|c|c|}
\hline \multirow[t]{2}{*}{$N$} & \multirow[t]{2}{*}{$\rho$} & \multirow[t]{2}{*}{$\tau_{1}^{2}$} & \multirow[t]{2}{*}{ Method } & \multicolumn{3}{|c|}{ NI-Intercept } & \multicolumn{3}{|c|}{ NI-Slope } & \multicolumn{3}{|c|}{ NI-Y3 } \\
\hline & & & & Bias & $\mathrm{SD}$ & Cov & Bias & SD & Cov & Bias & $\mathrm{SD}$ & $\mathrm{Cov}$ \\
\hline \multirow[t]{5}{*}{200} & -0.6 & $0 \cdot 25$ & $\mathrm{CC}$ & -254 & 68 & 14 & 219 & 70 & 82 & 136 & 68 & 394 \\
\hline & & & WLS & -22 & 75 & 886 & 7 & 75 & 886 & 100 & 64 & 570 \\
\hline & & & SMML & -43 & 69 & 822 & 17 & 72 & 864 & 97 & 68 & 582 \\
\hline & & & GLS & 22 & 75 & 878 & 9 & 75 & 886 & 117 & 64 & 470 \\
\hline & & & IML & -14 & 74 & 860 & 36 & 65 & 856 & 123 & 67 & 432 \\
\hline \multirow[t]{5}{*}{200} & 0.6 & $0 \cdot 25$ & $\mathrm{CC}$ & -65 & 33 & 756 & 215 & 69 & 70 & 206 & 70 & 100 \\
\hline & & & WLS & 69 & 70 & 748 & 4 & 74 & 896 & 136 & 70 & 390 \\
\hline & & & SMML & 66 & 66 & 764 & 28 & 77 & 826 & 142 & 66 & 304 \\
\hline & & & GLS & 49 & 73 & 824 & -3 & 73 & 894 & 111 & 71 & 496 \\
\hline & & & IML & 32 & 66 & 880 & 12 & 66 & 916 & 116 & 62 & 444 \\
\hline \multirow[t]{5}{*}{200} & -0.6 & $4 \cdot 0$ & $\mathrm{CC}$ & -544 & 165 & 48 & 975 & 138 & 0 & 916 & 130 & 0 \\
\hline & & & WLS & 81 & 151 & 876 & 66 & 150 & 888 & 213 & 142 & 604 \\
\hline & & & SMML & 81 & 158 & 852 & 92 & 154 & 874 & 212 & 143 & 566 \\
\hline & & & GLS & -19 & 148 & 902 & 25 & 150 & 904 & 127 & 142 & 808 \\
\hline & & & IML & -46 & 152 & 904 & 62 & 155 & 864 & 127 & 143 & 796 \\
\hline \multirow[t]{5}{*}{200} & 0.6 & $4 \cdot 0$ & $\mathrm{CC}$ & 209 & 176 & 676 & 985 & 136 & 0 & 1024 & 140 & 0 \\
\hline & & & WLS & 217 & 152 & 614 & 69 & 155 & 870 & 218 & 153 & 582 \\
\hline & & & SMML & 224 & 152 & 570 & 87 & 156 & 862 & 226 & 150 & 530 \\
\hline & & & GLS & 23 & 154 & 882 & 8 & 154 & 900 & 96 & 154 & 822 \\
\hline & & & IML & 11 & 162 & 892 & 26 & 155 & 888 & 104 & 153 & 796 \\
\hline \multirow[t]{5}{*}{1000} & -0.6 & $0 \cdot 25$ & $\mathrm{CC}$ & -252 & 29 & 0 & 214 & 31 & 0 & 136 & 33 & 4 \\
\hline & & & WLS & -23 & 32 & 842 & 7 & 33 & 908 & 99 & 30 & 64 \\
\hline & & & SMML & -51 & 32 & 494 & 27 & 32 & 764 & 100 & 30 & 32 \\
\hline & & & GLS & 21 & 32 & 858 & 9 & 33 & 898 & 117 & 30 & 12 \\
\hline & & & IML & -16 & 32 & 856 & 38 & 29 & 644 & 119 & 31 & 4 \\
\hline \multirow[t]{5}{*}{1000} & $0 \cdot 6$ & $0 \cdot 25$ & $\mathrm{CC}$ & -63 & 33 & 386 & 215 & 30 & 0 & 208 & 32 & 0 \\
\hline & & & WLS & 67 & 32 & 336 & 6 & 32 & 888 & 139 & 31 & 4 \\
\hline & & & SMML & 60 & 31 & 404 & 26 & 31 & 782 & 142 & 29 & 2 \\
\hline & & & GLS & 47 & 33 & 550 & -2 & 32 & 908 & 115 & 32 & 30 \\
\hline & & & IML & 32 & 32 & 716 & 13 & 31 & 864 & 121 & 29 & 4 \\
\hline \multirow[t]{5}{*}{1000} & -0.6 & $4 \cdot 0$ & $\mathrm{CC}$ & -535 & 77 & 0 & 989 & 58 & 0 & 905 & 62 & 0 \\
\hline & & & WLS & 92 & 71 & 622 & 76 & 69 & 728 & 195 & 66 & 114 \\
\hline & & & SMML & 89 & 71 & 662 & 92 & 70 & 670 & 215 & 65 & 42 \\
\hline & & & GLS & -9 & 70 & 906 & 33 & 69 & 864 & 107 & 67 & 546 \\
\hline & & & IML & -52 & 65 & 788 & 59 & 64 & 780 & 120 & 72 & 424 \\
\hline \multirow[t]{5}{*}{1000} & $0 \cdot 6$ & $4 \cdot 0$ & $\mathrm{CC}$ & 214 & 78 & 140 & 994 & 61 & 0 & 1015 & 60 & 0 \\
\hline & & & WLS & 216 & 66 & 52 & 76 & 67 & 714 & 214 & 63 & 62 \\
\hline & & & SMML & 220 & 68 & 68 & 97 & 71 & 636 & 226 & 69 & 52 \\
\hline & & & GLS & 19 & 68 & 888 & 16 & 67 & 912 & 907 & 64 & 632 \\
\hline & & & IML & -0 & 70 & 908 & 36 & 70 & 840 & 97 & 67 & 600 \\
\hline
\end{tabular}

$\rho=-0 \cdot 6, \tau_{1}^{2}=0 \cdot 25$ but exhibits substantial bias and poor coverage for other choices of parameters, particularly when $\rho=0 \cdot 6, \tau_{1}^{2}=4$. WLS is generally similar to SMML for these problems. Finally, CC is seriously biased and has very poor coverage in this setting.

(c) Results for the MAR-DIFF mechanism are broadly similar to results for the MAR-SUM mechanism. IML is again the best method, and GLS is competitive with IML for some choices of parameters but exhibits bias for others. SMML is comparable to IML when 
$\rho=0 \cdot 6, \tau_{1}^{2}=4$ but inferior to IML for other choices of parameters, where it is moderately biased and has inferior coverage. The bias of SMML is smaller for the MAR-DIFF mechanism than for the MAR-SUM mechanism. WLS is notably inferior to SMML for this set of simulations, and CC is again much worse than all the other methods in terms of bias and coverage.

(d) For the NI-Intercept mechanism, all the methods exhibit bias and below nominal coverage to some degree, but important differences between the methods emerge (Table IB). IML is the best method overall, with small or moderate bias and close to nominal coverage in all but one problem $\left(n=1000, \rho=0 \cdot 6, \tau_{1}^{2}=0 \cdot 25\right)$. GLS is similar and nearly as good as IML for these problems. SMML and WLS exhibit more bias and inferior coverage to IML and GLS, and CC is once again much the worst method in terms of bias and coverage.

(e) For the NI-Slope mechanism, IML and GLS are again the best methods, but GLS has somewhat lower bias and better coverage than IML for some problems $(n=1000$, $\left.\rho=-0 \cdot 6, \tau_{1}^{2}=0 \cdot 25\right)$. Further examination of the estimates indicates that IML tends to underestimate $\tau_{1}^{2}$ for these problems and GLS tends to overestimate $\tau_{1}^{2}$. These characteristics tend to lead to estimates of the slope that are less biased for GLS than for IML. SMML and WLS are similar and somewhat inferior to IML and GLS for these problems, and $\mathrm{CC}$ is again the worst method by a wide margin.

(f) For the NI-Y3 mechanism none of the methods is very satisfactory. IML and GLS again emerge as the best methods, but their coverage in the cases with sample size 1000 is very poor. CC is again the worst of the methods considered in terms of bias and coverage.

Table II displays bias and standard deviation of estimates of the between-subject variance $\tau_{1}^{2}$ from the five methods; the bias and precision of estimates of the within-subject variance $\sigma^{2}$ did not vary much between methods. GLS and WLS produce the same estimates of $\tau_{1}^{2}$ and hence have the same results in Table II. When data are missing completely at random, IML shows considerable gains in efficiency over alternative methods, with some evidence of bias that is diminished in the larger sample size problems. For other mechanisms, IML generally produces the best estimates of $\tau_{1}^{2}$, and the superiority of the maximum likelihood methods (IML, SMML) over method of moments counterparts (GLS, WLS) is evident.

\section{DISCUSSION}

Our simulation study includes a broader range of drop-out mechanisms and parameter values than previous studies. Nevertheless it is important to be cautious in generalizing conclusions, since no simulation can cover all the situations that arise in practice. With that caveat, a number of interesting findings emerge from our results.

A striking result is the very poor performance of $\mathrm{CC}$. This is in contrast with the simulations in Wang-Clow et al., ${ }^{24}$ where CC was very competitive with other methods. Those authors simulated a case where the variability of the slopes was very small. Our results underline the fact that $\mathrm{CC}$ can be very biased when data are not missing completely at random, and methods that use all the available data can reduce bias, improve precision and improve coverage in these settings.

Methods that use all the available information (IML and GLS) outperformed summary measures methods that discard the intercepts (SMML and WLS) when the mechanism was not missing completely at random, including both missing at random and non-ignorable cases. This is 
Table II. Bias and standard deviation of estimates of $\tau_{1}^{2}$ from five methods

\begin{tabular}{|c|c|c|c|c|c|c|c|c|c|c|c|c|c|c|c|}
\hline \multirow[t]{2}{*}{$N$} & \multirow[t]{2}{*}{$\rho$} & \multirow[t]{2}{*}{$\tau_{1}^{2}$} & \multirow[t]{2}{*}{ Method } & \multicolumn{2}{|c|}{ MCAR } & \multicolumn{2}{|c|}{ MAR-SUM } & \multicolumn{2}{|c|}{ MAR-DIFF } & \multicolumn{2}{|c|}{ NI-Intercept } & \multicolumn{2}{|c|}{ NI-Slope } & \multicolumn{2}{|c|}{ NI-Y3 } \\
\hline & & & & Bias & SD & Bias & SD & Bias & SD & Bias & SD & Bias & SD & Bias & SD \\
\hline \multirow[t]{5}{*}{200} & $-0 \cdot 6$ & 0.25 & $\mathrm{CC}$ & 3 & 107 & -88 & 88 & -28 & 90 & -94 & 96 & -45 & 94 & -30 & 111 \\
\hline & & & WLS & 15 & 173 & 279 & 184 & 348 & 162 & 210 & 176 & 162 & 186 & 6 & 169 \\
\hline & & & SMML & 18 & 107 & 62 & 116 & 138 & 132 & 24 & 111 & 41 & 116 & -10 & 97 \\
\hline & & & GLS & 15 & 173 & 279 & 184 & 348 & 162 & 210 & 176 & 162 & 186 & 6 & 169 \\
\hline & & & IML & 20 & 86 & 27 & 95 & 27 & 95 & -3 & 85 & -13 & 80 & -18 & 75 \\
\hline \multirow[t]{5}{*}{200} & $0 \cdot 6$ & $0 \cdot 25$ & $\mathrm{CC}$ & -10 & 110 & 12 & 94 & -31 & 91 & 26 & 106 & -63 & 97 & -78 & 98 \\
\hline & & & WLS & -5 & 167 & 216 & 171 & 353 & 159 & 135 & 166 & 182 & 157 & -3 & 165 \\
\hline & & & SMML & 24 & 110 & 105 & 120 & 129 & 118 & 74 & 109 & 32 & 113 & -68 & 92 \\
\hline & & & GLS & -5 & 167 & 216 & 171 & 353 & 159 & 135 & 166 & 182 & 157 & -3 & 165 \\
\hline & & & IML & 70 & 78 & 84 & 85 & 84 & 88 & 78 & 81 & 64 & 86 & 16 & 71 \\
\hline \multirow[t]{5}{*}{200} & -0.6 & $4 \cdot 0$ & $\mathrm{CC}$ & 21 & 550 & -354 & 496 & -1673 & 358 & -562 & 489 & -1926 & 331 & -1773 & 327 \\
\hline & & & WLS & 7 & 523 & 364 & 544 & 454 & 519 & 368 & 531 & 211 & 532 & -64 & 519 \\
\hline & & & SMML & 18 & 492 & 285 & 564 & 130 & 569 & 190 & 528 & -186 & 512 & -507 & 498 \\
\hline & & & GLS & 7 & 523 & 364 & 564 & 456 & 519 & 368 & 531 & 211 & 532 & -64 & 519 \\
\hline & & & IML & 4 & 297 & 9 & 501 & -22 & 513 & -101 & 460 & -146 & 447 & -344 & 494 \\
\hline \multirow[t]{5}{*}{200} & $0 \cdot 6$ & $4 \cdot 0$ & $\mathrm{CC}$ & -13 & 541 & 108 & 542 & -1672 & 360 & -65 & 530 & -1928 & 329 & -1950 & 320 \\
\hline & & & WLS & -23 & 524 & 174 & 530 & 447 & 533 & 2 & 513 & 247 & 562 & -44 & 543 \\
\hline & & & SMML & 0 & 493 & 51 & 495 & 119 & 526 & -75 & 471 & -219 & 530 & -682 & 525 \\
\hline & & & GLS & -23 & 524 & 174 & 530 & 467 & 533 & 2 & 513 & 247 & 542 & -46 & 543 \\
\hline & & & IML & -63 & 663 & 62 & 438 & 33 & 487 & 76 & 458 & -4 & 526 & -252 & 490 \\
\hline \multirow[t]{5}{*}{1000} & -0.6 & $0 \cdot 25$ & $\mathrm{CC}$ & -3 & 46 & -85 & 41 & -31 & 40 & -96 & 41 & -54 & 43 & -36 & 48 \\
\hline & & & WLS & -1 & 78 & 281 & 80 & 352 & 79 & 209 & 83 & 157 & 84 & -12 & 83 \\
\hline & & & SMML & -6 & 48 & 50 & 57 & 115 & 56 & 3 & 55 & 24 & 53 & -25 & 48 \\
\hline & & & GLS & -1 & 78 & 281 & 80 & 352 & 79 & 209 & 83 & 157 & 84 & -12 & 83 \\
\hline & & & IML & -5 & 39 & 8 & 39 & 12 & 37 & -10 & 33 & -30 & 35 & -33 & 35 \\
\hline \multirow[t]{5}{*}{1000} & $0 \cdot 6$ & 0.25 & $\mathrm{CC}$ & 2 & 46 & 13 & 45 & -33 & 40 & 28 & 50 & -55 & 44 & -76 & 42 \\
\hline & & & WLS & 2 & 77 & 214 & 78 & 352 & 80 & 116 & 82 & 159 & 81 & -8 & 81 \\
\hline & & & SMML & 6 & 49 & 95 & 54 & 115 & 55 & 65 & 50 & 24 & 51 & -58 & 48 \\
\hline & & & GLS & 2 & 77 & 214 & 78 & 352 & 80 & 116 & 82 & 159 & 81 & -8 & 81 \\
\hline & & & IML & 6 & 36 & 76 & 34 & 77 & 34 & 74 & 36 & 54 & 36 & 3 & 34 \\
\hline \multirow[t]{5}{*}{1000} & $-0 \cdot 6$ & $4 \cdot 0$ & $\mathrm{CC}$ & 7 & 239 & -346 & 221 & -1650 & 161 & -540 & 218 & -1933 & 143 & -1792 & 150 \\
\hline & & & WLS & -5 & 223 & 368 & 231 & 437 & 238 & 337 & 230 & 247 & 227 & -66 & 246 \\
\hline & & & SMML & -11 & 213 & 292 & 211 & 84 & 236 & 185 & 232 & -207 & 239 & -524 & 221 \\
\hline & & & GLS & -5 & 223 & 368 & 231 & 437 & 238 & 337 & 230 & 247 & 227 & -66 & 246 \\
\hline & & & IML & 24 & 216 & -11 & 211 & -32 & 233 & -125 & 205 & -197 & 213 & -371 & 227 \\
\hline \multirow[t]{5}{*}{1000} & 0.6 & $4 \cdot 0$ & $\mathrm{CC}$ & 5 & 249 & 28 & 256 & -1662 & 167 & -83 & 228 & -1931 & 145 & -1945 & 151 \\
\hline & & & WLS & -6 & 232 & 103 & 236 & 431 & 228 & -23 & 219 & 258 & 242 & -55 & 247 \\
\hline & & & SMML & -16 & 220 & 107 & 212 & 92 & 234 & -39 & 229 & -193 & 242 & -514 & 249 \\
\hline & & & GLS & -6 & 232 & 103 & 236 & 431 & 228 & -23 & 219 & 258 & 242 & -55 & 247 \\
\hline & & & IML & 30 & 192 & 22 & 210 & 58 & 213 & 57 & 216 & -100 & 211 & -217 & 206 \\
\hline
\end{tabular}

an important finding since the simplicity of methods based on the slopes alone is compelling, and it is generally thought that these methods involve minor losses of efficiency over full maximum likelihood. Our simulations suggest that this is indeed the case when the mechanism is missing completely at random, but not when missingness depends on the data.

A related point is that SMML is generally biased under missing at random mechanisms, as is seen in our simulations under MAR-SUM and MAR-DIFF and predicted by the theoretical argument in Section 2. SMML is a maximum likelihood method, but it is not valid under missing at random since it discards data, namely the intercepts. Further inspection of the results shows that the bias of SMML is generally greater for MAR-SUM and NI-Intercept mechanisms than 
for MAR-DIF and NI-Slope mechanisms. This finding reflects the fact that missingness is more closely associated with the estimated intercepts for MAR-SUM and NI-Intercept mechanisms than for the MAR-DIF and NI-Slope mechanisms. Thus dropping the information about the mechanism carried in the intercepts leads to a greater bias in those cases.

Note that under subject-specific missing at random, the estimated slope for each individual is a consistent estimate of the true slope for that individual as the number of repeated measures tends to infinity. The individual-level summary measures are valid in this weak sense for all the mechanisms in our study except NI-Y3, which is not subject-specific missing at random. However, the overall estimate of the slope in SMML is a weighted average of the individual slopes, with weights that depend on the estimate of $\tau_{1}^{2}$. It is bias in the estimate of $\tau_{1}^{2}$, as reflected in Table II, that creates the problem in the SMML estimate of $\theta_{1}$.

The bias of SMML for missing at random mechanisms does not necessarily mean that the method is always inferior to IML for non-ignorable mechanisms. We believe that it is theoretically possible for SMML to have less bias than IML under some circumstances, but we found no real evidence of this in our simulations. IML generally outperformed SMML for the nonignorable mechanisms we simulated.

We note that IML was noticeably superior to GLS in our missing at random simulations, reflecting the fact that maximum likelihood, unlike the method of moments, yields consistent estimates of variance under MAR-SUM and MAR-DIFF. For the non-ignorable mechanisms neither method consistently dominated the other. Overall the added computation of IML over GLS has little pay-off when the data are missing completely at random, but IML seems preferable to GLS when the data are not missing completely at random.

None of the methods considered here is satisfactory for all mechanisms, as indicated by the disappointing results for the NI-Y3 mechanism. As noted in the introduction, in these simulations we have not studied methods that are tuned to non-ignorable drop-out mechanisms. There is a growing literature of these methods, ${ }^{7-15}$ as discussed in Little. ${ }^{16}$ In their simulation study, Wang-Clow et al. $^{24}$ consider unweighted least squares (UWLS), which computes unweighted averages of the least squares slope of individuals in each treatment group. This method is unbiased for mechanisms that depend on the unobserved random slopes and intercepts, but the method had poor precision in their simulations. A more promising approach is the weighted least squares method considered by Wang-Clow et al. ${ }^{24}$ that incorporates a covariate adjustment on time to drop-out. This ANCOVA method performed well for the non-ignorable mechanisms in Wang-Clow et al., but was inferior to IML for missing at random mechanisms. Wang-Clow et $a .^{24}$ computed the variance components in this ANCOVA method using the method of moments, as in $\mathrm{Wu}$ and Bailey; ${ }^{8}$ since method of moments approaches did not do as well as maximum likelihood in our simulations, we suggest that a maximum likelihood version of the method ANCOVA based on the pattern-mixture model in Little ${ }^{16}$ might do even better.

Methods tuned to particular non-ignorable mechanisms yield consistent estimates under covariate-dependent drop-out, although there is a loss of efficiency of estimation that might be considerable. It is important to emphasize that these methods are in general biased under missing at random drop out, since the missing at random model is not generally a submodel of the hypothesized non-ignorable mechanism. No method has been found that performs uniformly well for the broad range of missing at random and not missing at random mechanisms that might occur in practice. Whether such methods can be found remains an open question. 


\section{ACKNOWLEDGEMENTS}

This research was supported by a grant from the National Science Foundation. We thank a referee for useful suggestions on an earlier draft.

\section{REFERENCES}

1. Harville, D. A. 'Maximum likelihood approaches to variance component estimation and to related problems (with discussion)', Journal of the American Statistical Association, 72, 320-340 (1977).

2. Laird, N. M. and Ware, J. H. 'Random-effects models for longitudinal data', Biometrics, 38, 963-974 (1982).

3. Jennrich, R. I. and Schluchter, M. D. 'Incomplete repeated-measures models with structured covariance matrices', Biometrics, 42, 805-820 (1986).

4. Dixon, W. J. BMDP Statistical Software, University of California Press, Berkeley, CA, 1988.

5. SAS 'The mixed procedure', in SAS/STAT Software: Changes and Enhancements, Release 6.07, Technical Report P-229, SAS Institute, Inc., Cary, NC, 1992, Chapter 16.

6. Rubin, D. B. 'Inference and missing data', Biometrika, 63, 581-592 (1976).

7. Wu, M. C. and Carroll, R. J. 'Estimation and comparison of changes in the presence of informative right censoring by modeling the censoring process', Biometrics, 44, 175-188 (1988).

8. Wu, M. C. and Bailey, K. R. 'Estimation and comparison of changes in the presence of informative right censoring: conditional linear model', Biometrics, 45, 939-955 (1989).

9. Schluchter, M. D. 'Methods for the analysis of informatively censored longitudinal data', Statistics in Medicine, 11, 1861-1870 (1992).

10. Little, R. J. A. 'Pattern-mixture models for multivariate incomplete data', Journal of the American Statistical Association, 88, 125-134 (1993).

11. Little, R. J. A. 'A class of pattern-mixture models for normal missing data', Biometrika, 81, (3), 471-483 (1994).

12. Little, R. J. A. and Wang, Y-X. 'Pattern-mixture models for multivariate incomplete data with covariates', Biometrics, 52, 98-111 (1996).

13. Diggle, P. and Kenward, M. G. 'Informative dropout in longitudinal data analysis (with discussion)', Applied Statistics, 43, 49-94 (1994).

14. Mori, M., Woolson, R. F. and Woodsworth, G. G. 'Slope-estimation in the presence of informative censoring: modeling the number of observations as a geometric random variable', Biometrics, 50, 39-50 (1994).

15. Molenberghs, G., Kenward, M. G. and Lesaffre, E. 'The analysis of longitudinal ordinal data with nonrandom dropout', Biometrika, 84, (1), 33-44 (1997).

16. Little, R. J. A. 'Modeling the drop-out mechanism in longitudinal studies', Journal of the American Statistical Association, 90, 1112-1121 (1995).

17. Dempster, A. P., Laird, N. M. and Rubin, D. B. 'Maximum likelihood from incomplete data via the EM algorithm (with discussion)', Journal of the Royal Statistical Society, Series B, 39, 1-38 (1977).

18. Little, R. J. A. and Rubin, D. B. Statistical Analysis with Missing Data, Wiley, New York, 1987.

19. Meng, X. L. and Van Dyk, D. 'The EM algorithm - an old folk song sung to a fast new tune, (with discussion)', Journal of the Royal Statistical Society, Series B, 59, (3), 511-568 (1997).

20. Gelfand, A. E. and Smith, A. F. M. 'Sampling-based approaches to calculating marginal densities', Journal of the American Statistical Association, 85, 398-409 (1990).

21. Tanner, M. A. Tools for Statistical Inference, 3rd edn, Springer-Verlag, New York, 1996.

22. Gilks, W. R., Wang, C. C., Yvonnet, B. and Coursaget, P. 'Random-effects models for longitudinal data using Gibbs' sampling', Biometrics, 49, 441-453 (1993).

23. Vonesh, E. F. and Carter, R. L. 'Efficient inferences for random-coefficient growth curve models with unbalanced data', Biometrics, 43, 617-628 (1987).

24. Wang-Clow, F., Lange, M., Laird, N. M. and Ware, J. H. 'Simulation study of estimators for rate of change in longitudinal studies with attrition', Statistics in Medicine, 14, $283-297$ (1995). 